\title{
CURVA DE SOBREVIVÊNCIA E ESTIMATIVA DE ENTROPIA EM LUCILIA CUPRINA (DIPTERA, CALLIPHORIDAE)
}

\author{
Francely M. Fernandes ${ }^{1}$ \\ David M. Lapola ${ }^{1}$ \\ Rodrigo Neregato ${ }^{1}$ \\ Marcelo H. de Carvalho ${ }^{1}$ \\ Claudio J. Von Zuben ${ }^{1}$
}

\begin{abstract}
SURVIVORSHIP CURVE AND ESTIMATE OF ENTROPY IN LUCILIA CUPRINA (DIPTERA, CALLIPHORIDAE). Lucilia cuprina (Wiedemann, 1830) is a cosmopolite blowfly species of medical and veterinary importance because it produces myiasis, mainly in ovine. In order to evaluate the demographic characteristics of this species, survivorship curves for 327 adult males and 323 adult females, from generation $F_{1}$ maintained under experimental conditions, were obtained. Entropy was utilized as the estimator of the survival pattern to quantify the mortality distribution of individuals as a function of age. The entropy values 0.216 (males) and 0.303 (females) were obtained. These results denote that, considering the survivorship interval until the death of the last individual for each sex, the males present a tendency of mortality in more advanced age intervals, in comparison with the females.
\end{abstract}

KEYWORDS. Survivorship curve, entropy, Lucilia, Calliphoridae.

\section{INTRODUÇÃO}

Os dípteros califorídeos são insetos de importância ecológica, veterinária e médicosanitária, pelo seu papel como decompositores de matéria orgânica, vetores mecânicos de patógenos, causadores de míases, além de serem utilizados como indicadores do intervalo pós-morte (IPM) em cadáveres humanos, em investigações médico-criminais (Zumpt, 1965; Furlanetto et al., 1984; Hanski, 1987; Guimarães \& PapaVero, 1999; Von Zuben, 2001). Lucilia cuprina (Wiedemann, 1830) é uma mosca-varejeira cosmopolita, muito estudada na Austrália, por causar miíases em ovelhas (NoRRIs, 1990; FENTON et al., 1999; Mariconi et al., 1999). Na África do Sul, mais de $90 \%$ das miíases em ovelhas são causadas por L. cuprina e, na Austrália, de 60 a 90\% dos casos (NorRIs, 1959).

1. Instituto de Biociências, Universidade Estadual Paulista, UNESP, Av. 24A, 1515, Bela Vista, 13506-900, Rio Claro, SP, Brasil. (vonzuben@rc.unesp.br) 
Estas moscas adultas são encontradas em lixo urbano, frutos caídos e néctar de flores (MARICONI et al., 1999). Lucilia cuprina prefere armadilhas com vísceras de galinha e carcaças de rato, mas também é encontrada sobre fezes humanas. É uma espécie eussinantrópica, de importância médico-sanitária, muito comum em lixões na região de Campinas e São Paulo (Linhares, 1981). Almeida \& Mello (1996) estudaram o comportamento de dípteros muscóides frente a substratos de oviposição em laboratório e observaram preferência das fêmeas de L. cuprina por carne bovina moída. As fêmeas necessitam ter uma alimentação proteica para conseguir a maturação dos ovos. A larva eclodida alimenta-se da linfa que extravasa do tecido epidérmico do hospedeiro, assim como do tecido necrótico das feridas e de carcaças de animais, durante o ano inteiro, com média de 9 a 10 gerações por ano (MARICONi et al., 1999).

O objetivo foi confeccionar curvas de sobrevivência e tabelas de vida para uma população experimental de L. cuprina. Calculou-se o valor da entropia (DemETRIUs, 1978; CAREY, 1993) para ambos os sexos, como estimador do padrão de sobrevivência, para quantificar a distribuição de mortalidade dos adultos em função da idade.

\section{MATERIAL E MÉTODOS}

Utilizou-se a primeira geração originada de postura de moscas capturadas no Instituto de Biociências, Universidade Estadual Paulista, Campus de Rio Claro, São Paulo. Como isca, foram utilizados peixes em decomposição. Os adultos foram capturados com o auxílio de um puçá em outubro de 2000, e acondicionados em gaiolas (30x30x40 cm) de armação de ferro e tela de náilon. Foram fornecidos às moscas, água, açúcar e carne bovina moída, como substrato de oviposição, e fígado bovino como complemento alimentar para a maturação dos ovos.

Os adultos emergidos foram acondicionados em duas gaiolas totalizando 650 indivíduos (327 machos e 323 fêmeas), mantidos em sala climatizada com temperatura de $25 \pm 1{ }^{\circ} \mathrm{C}, 60 \%$ de UR e fotoperíodo de 12 horas. Diariamente, foram realizadas a contagem e sexagem dos indivíduos mortos até o último indivíduo. Os espécimens foram depositados na Coleção Entomólogica do Departamento de Zoologia, Instituto de Biociências, Universidade Estadual Paulista, Campus de Rio Claro.

Para o estudo do padrão de sobrevivência, foram utilizadas tabelas de vida com a técnica apresentada por DemETRIUS (1978) e CAREY (1993), cujos parâmetros são necessários para a estimativa da entropia (Krebs, 1989; CAREY, 1993). Os parâmetros calculados foram os usuais para tabelas de vida (CAREY, 1993).

Os intervalos em dias correspondentes ao eixo x (figs. 1, 2) são diferentes, porque as últimas fêmeas viveram até 11 dias a mais que o último macho. As figuras foram feitas em Excel e o eixo x numerou em intervalos de 3 dias (fig. 1) e de 4 dias (fig. 2).

A partir dos dados da tabela de vida, foi calculada a entropia, que serve como um estimador quantitativo da curva de sobrevivência, possibilitando assim fazer uma comparação mais detalhada entre os padrões de sobrevivência de indivíduos dos dois sexos ou de espécies diferentes. Fórmula da entropia (H) segundo CAREY (1993) sendo $e_{0}$ a expectativa de vida na idade inicial da população, $d_{x}$ a proporção de indivíduos que morre entre as idades $x$ e $x+1, w$ a idade máxima em dias alcançada pelo último indivíduo sobrevivente e $\sum_{x=0}^{w} e_{x} d_{x}$ o somatório das médias ponderadas das esperanças de vida em todas as idades.

$$
\mathrm{H}=\sum_{x=0}^{w} e_{x} d_{x} / e_{0}
$$

Iheringia, Sér. Zool., Porto Alegre, 93(3):319-324, 30 de setembro de 2003 


\section{RESULTADOS E DISCUSSÃO}

Os valores dos parâmetros necessários para a estimativa de entropia são: $e_{0}=20,02$ dias, $w=45$ dias e $\sum_{x=0}^{w} e_{x} d_{x}=5,225$ (para machos); $e_{0}=29,48$ dias, $w=58$ dias e $\sum_{x=0}^{w} e_{x} d_{x}=8,932 \quad$ (para fêmeas).

Os valores de entropia $(\mathrm{H})$ obtidos para machos e fêmeas de L. cuprina, foram, respectivamente, 0,261 e 0,303 (figs.1,2).

Segundo CAREY (1993), quanto menor o valor da entropia, mais tarde os indivíduos tendem a morrer e quanto maior o valor, maior a mortalidade nos intervalos iniciais de tempo. Os valores extremos de entropia ocorrem, caso todos os indivíduos alcançarem a máxima longevidade fisiológica da espécie (DEMETRIUs, 1978; CAREY, 1993), sendo que o gráfico ilustrando a proporção de sobreviventes $l_{x}$ em função da idade $x$, teria um formato retangular $(\mathrm{H}=0)$. Quando $\mathrm{H}=1$, o padrão de sobrevivência caracteriza-se por alta mortalidade nos intervalos iniciais e uma expectativa de vida crescente com a idade dos sobreviventes. A sobrevivência decresce exponencialmente com a idade. Se todos os indivíduos tivessem exatamente a mesma probabilidade de morte em cada idade $x$ $(\mathrm{H}=0,5)$, ou seja, a mortalidade não depende da idade.

$\mathrm{O}$ valor de $e_{0}$ foi maior para as fêmeas do que para os machos de L. cuprina, sugerindo que o valor de entropia é menor para as fêmeas, já que $e_{0}$ aparece no denominador da expressão para o cálculo de entropia. Este cálculo depende da relação entre o somatório das médias ponderadas de expectativa de vida na idade $x$ e $e_{0}$, e não somente do valor de $e_{0}$. Considerando o intervalo de tempo de sobrevivência até o último indivíduo para cada sexo, os machos apresentam uma maior tendência de mortalidade nas idades mais avançadas, em comparação com as fêmeas.

Almeida \& Almeida (1996) construíram tabelas de vida considerando intervalos de semanas para adultos de L cuprina mantidos a $22-28^{\circ} \mathrm{C}$ em laboratório. A longevidade média foi de $6,1 \pm 2,6$ e $6,1 \pm 2,3$ semanas para machos e fêmeas, respectivamente. $O$ fato de não ter havido diferença significativa entre esses valores pode estar relacionado à grande variação de temperatura (de 22 a $28^{\circ} \mathrm{C}$ ) observada neste caso.

Os valores aqui encontrados para entropia foram diferentes daqueles observados por Von Zuben et al. (1996) para outro califorídeo, Chrysomya megacephala (Fabricius, 1794), mantido sob condições semelhantes. Os valores encontrados por esses autores para um experimento foram de 0,320 para machos e 0,287 para fêmeas. Comparando o presente estudo com o de Von Zuben et al. (1996), quando se considera o intervalo de tempo de sobrevivência até o último indivíduo para cada sexo, intervalo este que difere entre os sexos, os machos de L. cuprina e fêmeas de C. megacephala tendem a morrer mais tarde que os indivíduos do sexo oposto para cada espécie. Em estudos de sobrevivência, os indivíduos mais longevos ficam mais próximos da máxima longevidade fisiológica da espécie, ou seja, sua curva de sobrevivência aproxima-se mais da distribuição retangular.

KENNEDY \& HANCE (1995) estimaram a entropia para o ácaro plano Brevipalpus phoenicis (Geijskes, 1939) em diferentes variedades de chá do sul da India. Os valores de entropia obtidos variaram entre 0,120 (para indivíduos dos dois sexos na variedade de 


\section{Entropia - Machos $(\mathrm{H}=0,261)$}

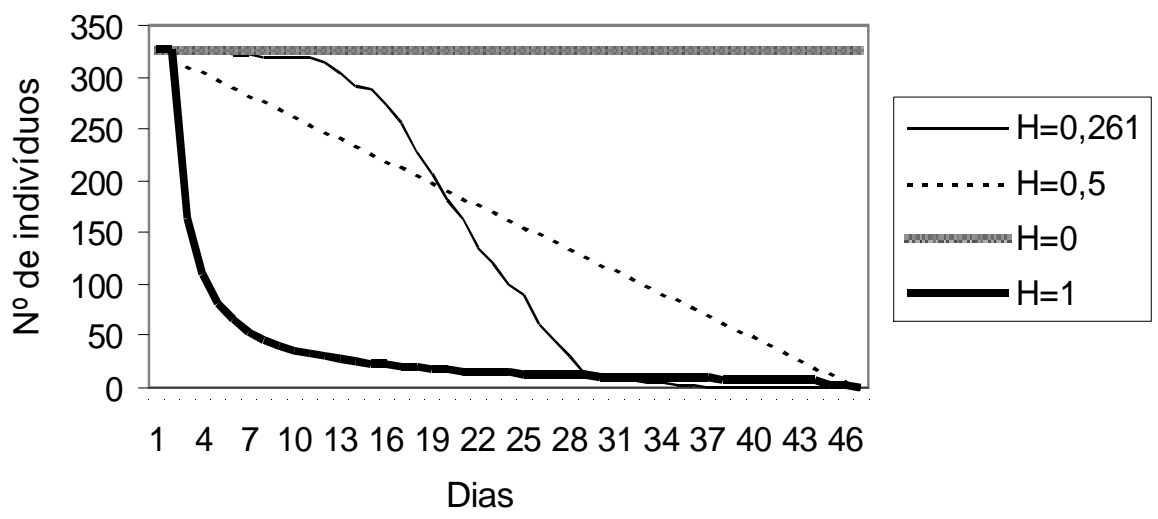

Fig 1. Curva de sobrevivência para machos de Lucilia cuprina, apresentando os valores teóricos de entropia $(\mathrm{H}=0 ; \mathrm{H}=0,5 ; \mathrm{H}=1)$.

\section{Entropia - Fêmeas $(\mathrm{H}=0,303)$}

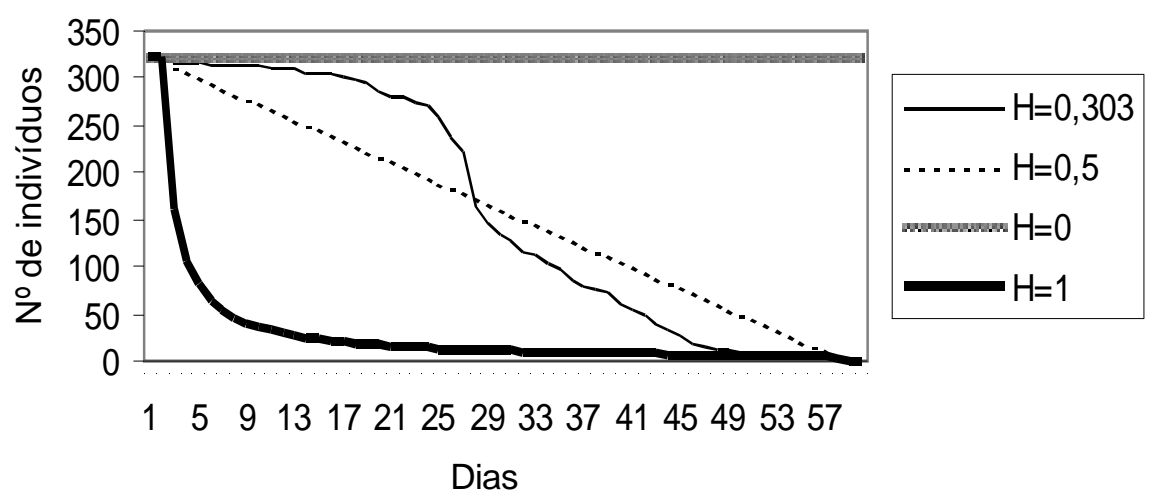

Fig 2. Curva de sobrevivência para fêmeas de Lucilia cuprina, apresentando os valores teóricos de entropia $(H=0 ; H=0,5 ; H=1)$. 
chá Shun mei) e 0,256 (na variedade Benihomare), indicando que os ácaros apresentam maior tendência de mortalidade em faixas etárias mais avançadas.

Da mesma forma que nos estudos citados anteriormente, os resultados obtidos no presente trabalho indicam que, para ambos os sexos de L. cuprina, há uma tendência dos valores de entropia aproximarem-se mais de zero do que da unidade, ou seja, pode-se observar uma tendência geral de mortalidade nas idades mais avançadas para ambos os sexos.

Quando se estuda aspectos de ecologia populacional de uma espécie, os dois principais parâmetros a serem considerados são sobrevivência e fecundidade, já que ambos interferem diretamente no tamanho populacional das próximas gerações (DE JoNG, 1982; HansKi, 1987; Von ZuBEN et al., 1993). Uma fêmea de mosca-varejeira com maior longevidade pode apresentar mais de um ciclo gonotrófico (LiNHAREs, 1988), o que lhe permite a realização de posturas em mais substratos (RoFf, 1977; DE JoNG, 1982). Isto normalmente aumenta a probabilidade de persistência local da espécie, porque o padrão de distribuição dos imaturos nos substratos alimentares, pelas fêmeas adultas, tem relação direta com o nível de competição por alimento que esses imaturos irão encontrar nestes substratos, com conseqüências para a viabilidade dos adultos resultantes (DE JonG, 1982; Von Zuben et al., 1996, 2001).

\section{REFERÊNCIAS BIBLIOGRÁFICAS}

Almeida, J. M. D' \& Almeida, J. R. 1996. Longevidade e curva de sobrevivência de oito espécies de dípteros caliptrados (Calliphoridae, Muscidae e Sarcophagidae), em condições de laboratório. Revta bras. Biol., São Carlos, 56(3):497-505.

Almeida, J. M. D' \& Mello, R. P. 1996. Comportamento de dípteros muscóides frente a substratos de oviposição, em laboratório, no Rio de Janeiro, RJ, Brasil. Mems Inst. Oswaldo Cruz, Rio de Janeiro, 91(1):131-136.

Carey, J. R. 1993. Applied demography for biologists. New York, Oxford University. 206p.

De Jong, G. 1982. The influence of dispersal pattern on the evolution of fecundity. Neth. J. Zool., Leiden, 32(1):1-30.

Demetrius, L. 1978. Adaptative value, entropy and survivorship curve. Nature, London, 275:213, 214 .

Fenton, A.; Wall, R. \& French, N. P. 1999. Oviposition aggregation by the blowfly Lucilia cuprina. Med. Vet. Entomol., Oxford, 13:453-456.

Furlanetto, S. M. P.; Campos, M. L. C. et al. 1984. Microrganismos enteropatogênicos em moscas africanas pertencentes ao gênero Chrysomya (Diptera: Calliphoridae) no Brasil. Revta Microbiol., São Paulo, 15(3):170-174.

Guimarães, J. H. \& Papavero, N. 1999. Myiasis in man and animals in the Neotropical region. São Paulo, Plêiade. 308p.

HANSKI, I. 1987. Carrion fly community dynamics: patchiness, seasonality and coexistence. Ecol. Entomol., Oxford, 12:257-266.

Kennedy, J. S. \& HANCE, T. 1995. Varietal screening based on demographic parameters: Resistance of tea to Brevipalpus phoenicis (Acari: Tenuipalpidae). Environ. Entomol., Lanham, 24:1481-1486.

KREBS, C. J. 1989. Ecological methodology. Cambridge, Harper \& Row. 654p.

LinHARES, A. X. 1981. Synanthropy of Calliphoridae and Sarcophagidae (Diptera) in the city of Campinas, São Paulo, Brazil. Revta bras. Ent., São Paulo, 25(3):189-215.

1988. The gonotrophic cycle of Chrysomya megacephala (Diptera: Calliphoridae) in the laboratory. Revta bras. Ent., São Paulo, 32(3/4):383-392.

Mariconi, F. A. M.; Guimarães, J. H. \& Filho, E. B. 1999. A mosca doméstica e algumas outras moscas nocivas. Piracicaba, FEALQ. 135p. 
Norris, K. R. 1959. The ecology of sheep blowflies in Australia. Monog. Biol., Denhaag, 8:514-544.

1990. Evidence for the multiple exotic origin of Australian populations of the sheep blowfly, Lucilia cuprina (Wiedemann) (Diptera: Calliphoridae). Aust. J. Zool., Collingwood, 38:635-648.

RofF, D. 1977. Dispersal in dipterans: its costs and consequences. J. Anim. Ecol., Oxford, 46:443-456.

Von Zuben, C. J. 2001. Zoologia aplicada: recentes avanços em estudos de entomologia forense. Entomol. Vec., Rio de Janeiro, 8(2):173-183.

Von Zuben, C. J.; Godoy, W. A. C. \& Monteiro Filho, E. L. A. 1996. Curva de sobrevivência e estimativa de entropia em Chrysomya megacephala (Diptera, Calliphoridae). Revta bras. Ent., São Paulo, 40(2):221-224.

Von Zuben, C. J.; Reis, S. F. et al. 1993. Dynamics of a mathematical model of Chrysomya megacephala (Diptera, Calliphoridae). J. Med. Entomol., Lanham, 30(2):443-448.

Von Zuben, C. J.; Von Zuben, F. J. \& Godoy, W. A. C. 2001. Larval competition for patchy resources in Chrysomya megacephala (Dipt., Calliphoridae): implications of the spatial distribution of immatures. J. Appl. Ent., Freising, 125:537-541.

Zumpt, F. 1965. Myiasis in man and animals in the Old World. London, Butterworths. $267 \mathrm{p}$.

Recebido em 11.06.2002; aceito em 01.07.2003.

Iheringia, Sér. Zool., Porto Alegre, 93(3):319-324, 30 de setembro de 2003 
